\title{
Die schweizerischen Banken und die Schuldenkrise der Dritten Welt
}

Les banques suisses et la crise d'endettement des pays du Tiers Monde

Georg Junge

\section{(2) OpenEdition}

1 Journals

\section{Electronic version}

URL: http://journals.openedition.org/sjep/1061

DOI: 10.4000/sjep.1061

ISSN: 1663-9677

\section{Publisher}

Institut de hautes études internationales et du développement

\section{Printed version}

Date of publication: 1 janvier 1986

Number of pages: 143-158

ISSN: 1660-5926

\section{Electronic reference}

Georg Junge, "Die schweizerischen Banken und die Schuldenkrise der Dritten Welt », Schweizerisches Jahrbuch für Entwicklungspolitik [Online], 6 | 1986, Online erschienen am: 09 März 2013, abgerufen am 08 September 2020. URL : http://journals.openedition.org/sjep/1061 ; DOI : https://doi.org/10.4000/ sjep. 1061 


\title{
DIE SCHWEIZERISCHEN BANKEN UND DIE SCHULDENKRISE DER DRITTEN WELT
}

\author{
Georg Junge, Schweizerischer Bankverein *
}

Résumé: Les banques suisses et la crise d'endettement des pays du Tiers Monde

La crise financière que le Mexique a connue en 1982 a provoqué une opération de sauvetage internationale de grande envergure. Le Mexique et une vingtaine d'autres pays débiteurs ont dû rééchelonner leurs dettes privées et publiques. Seule la concertation des actions a permis d'éviter l'effondrement des relations de crédit internationales. Grâce aux accords de rééchelonnement et à l'octroi de nouveaux prêts, les pays débiteurs ont pu procéder à certains ajustements qui ont apporté une amélioration passagère (jusqu'en 1985), quoique au prix de coûts sociaux élevés pour la population. En fait, d'un point de vue général, la situation s'est à nouveau dégradée en 1985. Les banques suisses ont adopté une attitude plus réservée que les autres pays en ce qui concerne l'octroi de crédits aux pays du Tiers Monde; extrêmement actives sur le plan des transactions inter-bancaires, elles sont en mesure de procurer des capitaux à court

*) Die in diesem Artikel vertretenen Meinungen sind diejenigen des Autors und entsprechen nicht unbedingt der Position des Schweizerischen Bankvereins. 
terme aux banques commerciales internationales. Une crise financière internationale affecterait donc les banques suisses avant tout sur ce plan-là. D'une manière générale, les banques suisses ont réagi favorablement à la proposition du ministre des finances américain, M. Baker, qui consiste à intensifier la capacité de croissance de quinze pays en développement fortement endettés. En outre, les banques suisses ont noté avec satisfaction que le gouvernement américain semblait reconnaître que la crise internationale de l'endettement revêt aussi un aspect politique. Le plan Baker prévoit notamment d'augmenter les crédits accordés par les banques commerciales aux pays débiteurs. Les banques suisses ont pris position à plusieurs reprises, réclamant une plus grande latitude pour ce qui a trait aux négociations sur le rééchelonnement des dettes. En effet, la pratique actuelle qui consiste à apporter une contribution financière uniquement sous forme d'argent frais, est ressentie par les banques comme une contrainte inutile. A leur avis, une option possible serait d'accorder un moratoire sur les intérêts. Par ailleurs, les banques aimeraient pouvoir attribuer davantage de crédits commerciaux, car ils sont importants pour les échanges commerciaux des pays en voie de développement et offrent davantage de garanties que les simples crédits financiers. Les banques admettent qu'un système efficace de relations de crédits n'entraîne pas nécessairement un transfert de ressources vers les pays en voie de développement, ce qui d'ailleurs apparaît souhaitable en vertu des critères de politique du développement. L'auteur conclut son article en énumérant les points qui lui paraissent essentiels dans le présent contexte: le capital prêté doit être évalué au cours du marché et remboursé comme tel; les pays débiteurs sont tenus de mener une politique économique cohérente; l'aide officielle au développement doit combler les déficits financiers qui subsistent encore. 
Im Sommer 1982 meldete Mexiko ernste Schwierigkeiten bei der Erfüllung seiner Zahlungsverpflichtungen an. Aber Mexiko war nur die Spitze eines Eisberges von rund 20 Ländern, die in den folgenden Wochen um Restrukturierung ihrer Auslandsschuld oder um Zahlungserleichterungen nachsuchten. Die plötzlichen Zahlungsschwierígkeiten der Länder stellten nicht nur eine ernsthafte Bedrohung der betroffenen Wirtschaften dar, sondern gefährdeten in ihrer Häufung auch das internationale Finanzsystem.

Um die rasch um sich greifende internationale Schuldenkrise unter Kontrolle zu bringen, waren koordinierte Anstrengungen der Schuldnerländer, der privaten und staatlichen Gläubiger und der internationalen Finanzinstitutionen, insbesondere des Internationalen Währungsfonds (IWF) und der Weltbank, erforderlich. Ziel der Anstrengungen war es, die Welt vor einem Zusammenbruch des internationalen Handels- und Zahlungsmechanismus mit seinen negativen Folgen auf das Weltwirtschaftswachstum und das internationale Finanzsystem zu bewahren. Dazu waren in der Regel als erste Massnahme Beistands- und Ueberbrückungskredite notwendig, um die drohende Zahlungsunfähigkeit einzelner Länder zu verhindern. Diese Beistandshilfen wurden unter der Regie des IWF und der Bank für Internationalen Zahlungsausgleich (BIZ) organisiert. Daran schlossen sich die Umschuldung fälliger Zahlungsverpflichtungen sowie die Organisation von Finanzkrediten durch die privaten und öffentlichen Gläubiger an. Gleichzeitig leiteten die Schuldnerländer gewöhnlich in Abstimmung mit dem IWF wirtschaftspolitische Anpassungsmassnahmen ein.

Krisenmanagement kann aber kein permanenter Zustand sein. Vier Jahre nach Ausbruch der Mexiko-Krise zeigen sich sowohl bei den Schuldnern als auch bei den Gläubigern deutliche Ermüdungserscheinungen. Auf der Tagung der Bretton-Woods-Institutionen in Seoul im Herbst 1985 hat der amerikanische Finanzminister darauf reagiert und den Anstoss für ein Ueberdenken der bisherigen Strategie gegeben.

In den folgenden Ausführungen wird zunächst in Abschnitt 1 das Krisenmanagement zwischen 1983 und 1985 erläutert sowie die Exposure der schweizerischen Banken im Kontext mit den anderen Bankengruppen dargestellt. Abschnitt 2 stellt die wichtigsten Elemente der Baker-Initiative vor und erläutert die Ueberlegungen der schweizerischen Banken dazu.

\section{DAS KRISENMANAGEMENT BIS 1985}

\subsection{Umfang der Umschuldungen}

Seit Mitte 1982 waren Umschuldungen und unfreiwillige Kreditvergabe die beiden Hauptinstrumente, die den Zusammenbruch der internationalen Kreditbeziehungen mit den Problemländern der Dritten Welt verhinderten. Als unfreiwillig wird hierbei jene Kreditvergabe bezeichnet, die im Rahmen von Umschuldungsabkommen gewährleistet wird. Die Banken sehen sich zu dieser Kreditvergabe neben der Umschuldung der Kapitalfälligkeiten ver- 
pflichtet, um die Zahlungsfähigkeit des Schuldners aufrechtzuerhalten. Diese unfreiwillige oder auch konzertierte Kreditvergabe steht im Gegensatz zu der freiwilligen bzw. spontanen Kreditvergabe an kreditwürdige Kunden, die ihren Finanzbedarf auf freien Kredit-und Kapitalmärkten erwerben. Tabelle 1 zeigt den Umfang der Umschuldungen fälliger Zahlungsverpflichtungen von zirka 35 Entwicklungsländern gegenüber offiziellen Gläubigern und Banken. Demnach vereinbarten zwischen 1983 und 1985 offizielle Gläubiger (im wesentlichen die Industriestaaten im Rahmen des Pariser Klubs) mit diesen Entwicklungsländern multilaterale Umstrukturierungen von Kapital- und Zinsfälligkeiten in Höhe von rund 23 Mrd. US-Dollar. Gleichzeitig haben die Geschäftsbanken mittel- und langfristige Kapitalfälligkeiten in der Grössenordnung von 145 Mrd. US-Dollar mit diesen Ländern umgeschuldet. Ausserdem wurden auf der Basis von Umstrukturierungsabkommen kurzfristige Kreditlinien in Höhe von 105 Mrd US-Dollar fortgeschrieben oder in mittelfristige Verbindlichkeiten umgewandelt. Schliesslich haben die Banken in den Jahren 1983 bis 1985 konzertierte Neuausleihungen in Höhe von 27 Mrd. US-Dollar getätigt, wobei diese Summe die Beteiligung an Ueberbrückungskrediten nicht mit einschliesst. Die schweizerischen Banken haben sich an diesen Umschuldungen und Neuausleihungen, wie jede andere nationale Bankengruppierung, nach Massgabe der bestehenden Engagements beteiligt.

Umschuldungen dieser Art können ihrer Natur nach nur temporären Charakter haben. Sie dienen dazu, vorübergehende Liquiditätsprobleme des Schuldners zu überbrücken und einen kontrollierten Anpassungsprozess zu ermöglichen. Gleichzeitig geben sie übermässig exponierten Banken die Gelegenheit, eine ausreichende Risikovorsorge zu treffen. Der kurzfristige Erfolg der Umschuldungen birgt allerdings die Gefahr der Gewöhnung in sich und kann auf die Dauer zu Lasten einer langfristigen Stabilisierung gehen. Sich ständig wiederholende Umschuldungen sind keine angemessene Reaktion auf langfristige strukturelle Probleme der Schuldnerländer und bestehende systematische Verzerrungen in den internationalen Kreditbeziehungen. Im Gegenteil, mit der Zeit vertiefen sie die existierenden Probleme. Auf Seiten der Schuldnerländer können sie die unerlässlichen Anpassungen unnötig hinauszögern, während gleichzeitíg das Engagement der Banken steigt, und möglicherweise neue Kreditgeber abgeschreckt werden. Mit jeder neuen Umschuldungsrunde steigt der Anreiz für solide Banken, aus diesem Karussell auszusteigen, während die Abhängigkeit der verbleibenden Banken vom Schuldnerland zunimmt.

In einer Situation, wo die Zahlungsprobleme eines Landes nicht nur temporärer Natur sind, wie die Umschuldungen unterstellen, sondern zum Teil auch längerfristige Probleme reflektieren, bedeuten die Umschuldungen zudem eine Vertagung der Wertberichtigung für den Teil der Kredite, der tatsächlich notleidend ist, während die Kreditwürdigkeit des Schuldners von Umschuldungsrunde zu Umschuldungsrunde verschlechtert wird. Die vernünftige und effiziente Lösung, die sich in diesem Falle anbietet, bestände in der Abgrenzung und Realisierung der bestehenden Verluste und der For- 


\section{TABELLE 1}

Umstrukturlerungen und "roll-over" der Schulden der Entwicklungsländer gegenüber Banken und offentlichen Stellen, In Mio. US \$ (1)

Banken (2)

Oeffentliche

Stellen (3)

\begin{tabular}{|c|c|c|}
\hline $\begin{array}{l}\text { Umstrukturlerung } \\
\text { von mittel- und } \\
\text { langfristlgen } \\
\text { Schulden }\end{array}$ & $\begin{array}{l}\text { Roll-over kurzfrl- } \\
\text { stiger Verbindlich- } \\
\text { kelten oder Umwand- } \\
\text { lung In mittelfrlstige } \\
\text { Verbindlichkeiten }\end{array}$ & $\begin{array}{l}\text { Multilaterale } \\
\text { Schuldenum- } \\
\text { strukturlerungen }\end{array}$ \\
\hline
\end{tabular}

1) IWF-Mitgliedsländer, d.h. ohne Polen; ohne Ueberbrückungskredite.

2) Schätzungen des IWF, Occasional Paper 43, Tabellen 37 bis 39.

3) Schátzungen des Institute of International Finance (IIF), Umschuldung von Kapital- und Zinsfälligkeiten.

mulierung von neuen Kreditverträgen, die an die wirtschaftliche Leistungsfähigkeit der-Schuldner anknüpfen (1).

\subsection{Die Anpassungsanstrengungen der Länder der Dritten Welt}

Die Anpassungserfolge, die die Länder der Dritten Welt seit 1982 erzielten, sind eindrücklich. Ein guter Tndikator dafür ist das Verhältnis zwischen dem Ertragsbilanzdefizit und den Exporteinnahmen. Für die 15 hochverschuldeten Baker-Länder (2) fiel dieses Verhältnis von 35\% im Jahre 1982 unter $1 \%$ im Jahre 1984 (3). Ein anderer signifikanter Indikator für die erfolgreiche Anpassung ist die Entwicklung der Handelsbilanzen. Während 1982 die aggregierte Handelsbilanz der 15 Baker-Länder einen Ueberschuss von nur 
3 Mrd. US-Dollar auswies und somit die Zinsverpflichtungen in Höhe von rund $46 \mathrm{Mrd}$. US-Dollar im wesentlichen durch Neugeld finanziert werden mussten, erzielten diese Länder 1984 einen Handelsbilanzüberschuss von 44 Mrd. US-Dollar und haben damit einen wesentlich grösseren Anteil ihrer Zinsverpflichtungen aus selbst erwirtschafteten Einnahmen finanzieren können. Allerdings hat sich 1985 die Lage der Länder erneut verschlechtert.

Die beeindruckenden externen Anpassungserfolge waren aber häufig nicht von wirtschaftlichen Erfolgen an der Innenfront begleitet. Die Versäumnisse der Vergangenheit in Form einer falsch ausgerichteten Geldund Fiskalpolitik und die Gewohnheit, systematisch mehr auszugeben als an Volkseinkommen zu erwirtschaften, mussten im Gefolge der eingeleiteten Austeritätspolitiken mit hohen Wachstumseinbussen teuer bezahlt werden. Das aggregierte Pro-Kopf-Einkommen der Länder der Dritten Welt schrumpfte stark und ist heute unter dem Niveau von 1980. Dieser Rückgang unterschätzt aber noch systematisch die tatsächlichen sozialen Anpassungskosten für die Bevölkerung. Die erwähnten Erfolge bei der Sanierung der Handels- und Ertragsbilanzen implizieren eine starke Reduktion der inländischen Absorption, d.h. der Konsum- und Investitionsausgaben sowie der staatlichen Sozialleistungen. Dadurch haben sich die Lebensbedingungen der Bevölkerung und das Investitionsklima in diesen Ländern stark verschlechtert, und dies führt zunehmend zu sozialen und politischen Spannungen und stellt auf die Dauer die politische Stabilität der betroffenen Regierungen in Frage.

\subsection{Die Risikovorsorge der Banken}

Ohne Umschuldungen und konzertierte Neuausleihungen wären zumindest zu Beginn der Schuldenkrise krisenhafte Abläufe denkbar gewesen, die auch verschiedene Banken in grosse Schwierigkeiten hätten bringen können. Insbesondere einige amerikanische Institute waren stark exponiert. Beispielsweise hatte Ende 1982 Manufacturers Hanover in den beiden Problemländern Brasilien und Mexiko ein Kreditengagement in Höhe von 144\% des Kapitals ausstehen, gefolgt von Citibank mit 128\% und Chemical Bank mit $112 \%(4)$.

Inzwischen haben die Banken die Zeit für eine erhöhte Risikovorsorge genutzt. Tabelle 2 zeigt, wie sehr sich zwischen 1982 une 1985 die Kredit/ Eigenkapitalquoten der US-Banken, das ist das Verhältnis von Kreditengagement zu den eigenen Mitteln, gegenüber dem Hauptschuldnerland Mexiko verbessert haben. Citicorp konnte seine Kredit/Eigenkapitalquote von $62 \%$ auf $25 \%$ reduzieren. Bei Citibank beruht die Verbesserung der Exposure einerseits auf einer absoluten Reduktion des Mexikoengagements von 3,4 Mrd. US-Dollar im Jahre 1982 auf 2,8 Mrd. US-Dollar im Jahre 1985 und andererseits auf einer kräftigen Erhöhung der Kapitalbasis. Bei in etwa konstantem Engagement haben auch die anderen Grossbanken der USA ihre Kapitalbasis deutlich verbessert, so dass sie im Vergleich zu 1982 heute weniger anfällig gegenüber Kreditausfällen geworden sind. 
TABELLE2

\section{Dle Mexiko-Position der grőssten US-Banken per \\ Ende 1982 und 1985 (1)}

\begin{tabular}{|c|c|c|c|c|}
\hline Bank & $\begin{array}{l}\text { Kreditaus } \\
\text { stand } \\
1982 \\
\text { Mrd. US \$ }\end{array}$ & $\begin{array}{l}\text { In \% des } \\
\text { Kapltals }\end{array}$ & $\begin{array}{l}\text { Kredltaus- } \\
\text { stand } \\
1985 \\
\text { Mrd. US \$ }\end{array}$ & $\begin{array}{l}\text { In } \% \text { des } \\
\text { Kapltals }\end{array}$ \\
\hline
\end{tabular}

\begin{tabular}{|c|c|c|c|c|}
\hline Citicorp & 3,4 & 62 & 2,9 & 25 \\
\hline Bank of America & 2,5 & 51 & 2,7 & 37 \\
\hline Manufacturers Hanover & 1.7 & 52 & 1,8 & 37 \\
\hline Chase Manhattan & 1,4 & 37 & 1,7 & 28 \\
\hline Chemical & 1,5 & 62 & 1,5 & 36 \\
\hline Bankers Trust & 0.8 & 48 & 1,3 & 39 \\
\hline Morgan Guaranty & 1,1 & 33 & 1,1 & 21 \\
\hline First Chicago & 0.8 & 47 & 0.9 & 32 \\
\hline
\end{tabular}

1) Quelle: Keefe, Bruyette \& Woods gemäss Neue Zürcher Zeitung vom 12. Juni $1986, S .17$.

Tabelle 3 zeigt, dass die markante Verbesserung der Kredit/Eigenkapitalquoten für den gesamten US-Bankensektor gilt und nicht auf Mexiko beschränkt blieb. Die Exposure fiel in allen Regionen der Dritten Welt (ohne Berücksichtigung von Osteuropa und dem Mittleren Osten) von 179\% der eigenen Mittel im Jahre 1982 auf 109\% im Jahre 1985. In der Problemregion Lateinamerika reduzierte sich die aggregierte Kredit/Eigenkapitalquote sogar um gut $1 / 3$ von $117 \%$ auf $77 \%$.

Die Zahlen der schweizerischen Banken sind von einem ausserordentlich vorsichtigen "Banking» charakterisiert (Tabelle 3). Selbst Ende 1982 machte der Gesamtumfang des Länderengagements in der Dritten Welt nur etwa $65 \%$ der eigenen Mittel aus. In Lateinamerika betrug die Kredit/Eigenkapitalquote rund $38 \%$. Angesicht dieser Zurückhaltung war ein Korrekturbedarf der schweizerischen Exposure nach 1982 kaum nötig (5). In der Tat hatten die schweizerischen Banken ihre Exposure in der Problemregion Lateinamerika bereits vor 1982 reduziert. Die Kredit/Eigenkapitalquote betrug dort 1979 $54 \%, 1980$ wurde ein einmaliger Spitzenwert von $62 \%$ verzeichnet, während 1981 mit 41\% bereits die Korrektur eintrat. Im Vergleich zu der Exposure der US-Banken waren dies immer noch sehr vorsichtige Engagements (6). Zieht 
man zudem Wertberichtungen und stille Reserven in Betracht, so dürtten die schweizerischen Banken jederzeit in einer international einzigartig guten Position gewesen sein.

Von der zurückhaltenden schweizerischen Kreditvergabe in der Dritten Welt zeugt auch Tabelle 4, die die geographische Verteilung der Engagements der schweizerischen Banken, der BIZ-Banken (7) sowie der US- und UKBanken per Ende 1984 zeigt. Sowohl im Vergleich zu den BIZ-Banken als zu den Vereinigten Staaten und dem Vereinigten Königreich waren die schweizerischen Banken in allen Regionen der Dritten Welt weniger exponiert. Die Guthaben in den sogenannten Baker-Ländern machten nur 6,6\% der gesamten schweizerischen Austandsguthaben aus, während das Engagement der BIZ-Banken in etwa doppelt so hoch war und die Vereinigten Staaten sogar $28 \%$ und das Vereinigte Königreich $23 \%$ ihrer Auslandsguthaben in diesen Ländern liegen hatten.

Auch diese Daten belegen, dass das direkte Engagement der schweizerischen Banken in der Dritten Welt verhältnismässig gering ist. Die internationale Verschuldungskrise dürfte somit für die schweizerischen Banken zu keinem Zeitpunkt eine signifikante direkte Gefährdung dargestellt haben. Demgegenüber hätten mögliche Sekundärwirkungen der Krise, etwa in Form von Solvenzkrisen einzelner grosser internationaler Banken oder eine eigentliche internationale Finanzkrise die schweizerischen Banken wahrscheinlich verhältnismässig härter getroffen, da ein Schwerpunkt ihrer Geschäftstätigkeit die Versorgung der grossen internationalen Geschäftsbanken mit kurzfristigem Kapital ist. Glücklicherweise konnte eine krisenhafte Ausweitung der internationalen Schuldenproblematik dank der Anstrengungen aller beteiligten Parteien abgewendet werden.

Selbstverständlich haben auch die Geschäftsbanken anderer Industrieländer und andere private und öffentliche Gläubiger die Zeit nach dem Ausbruch der Mexikokrise für eine erhöhte Risikovorsorge genutzt. Von den Banken der Bundesrepublik Deutschland ist bekannt, dass sie auf Konzernebene, also unter Einschlus ihrer Tochtergesellschaften 1982 in Lateinamerika mit $100 \%$ ihres Eigenkapitals engagiert waren, seitdem aber starke Anstrengungen in Form von Wertberechtigungen und Rückstellungen vorgenommen haben. Ein ähnliches Engagement und Verhalten lässt sich für die Geschäftsbanken in Grossbritannien zeigen (8).

Mit der Erhöhung der Risikovorsorge der Geschäftsbanken und anderen privaten und öffentlichen Kreditgebern gingen unvermeidlich die Ausleihungen in die Entwicklungsländer stark zurück. Nach Schätzungen der Weltbank fiel die Kreditvergabe (new loan commitments) an die Dritte Welt von insgesamt 103,0 Mrd. US-Dollar im Jahre 1981 auf 69,9 Mrd. US-Dollar im Jahre 1984. Am stärksten war der Einbruch bei den hochverschuldeten Ländern, wo in diesem Zeitraum ein Rückgang der Kreditvergabe von $79 \mathrm{Mrd}$. US-Dollar auf 29,8 Mrd. US-Dollar zu verzeichnen war (9). 

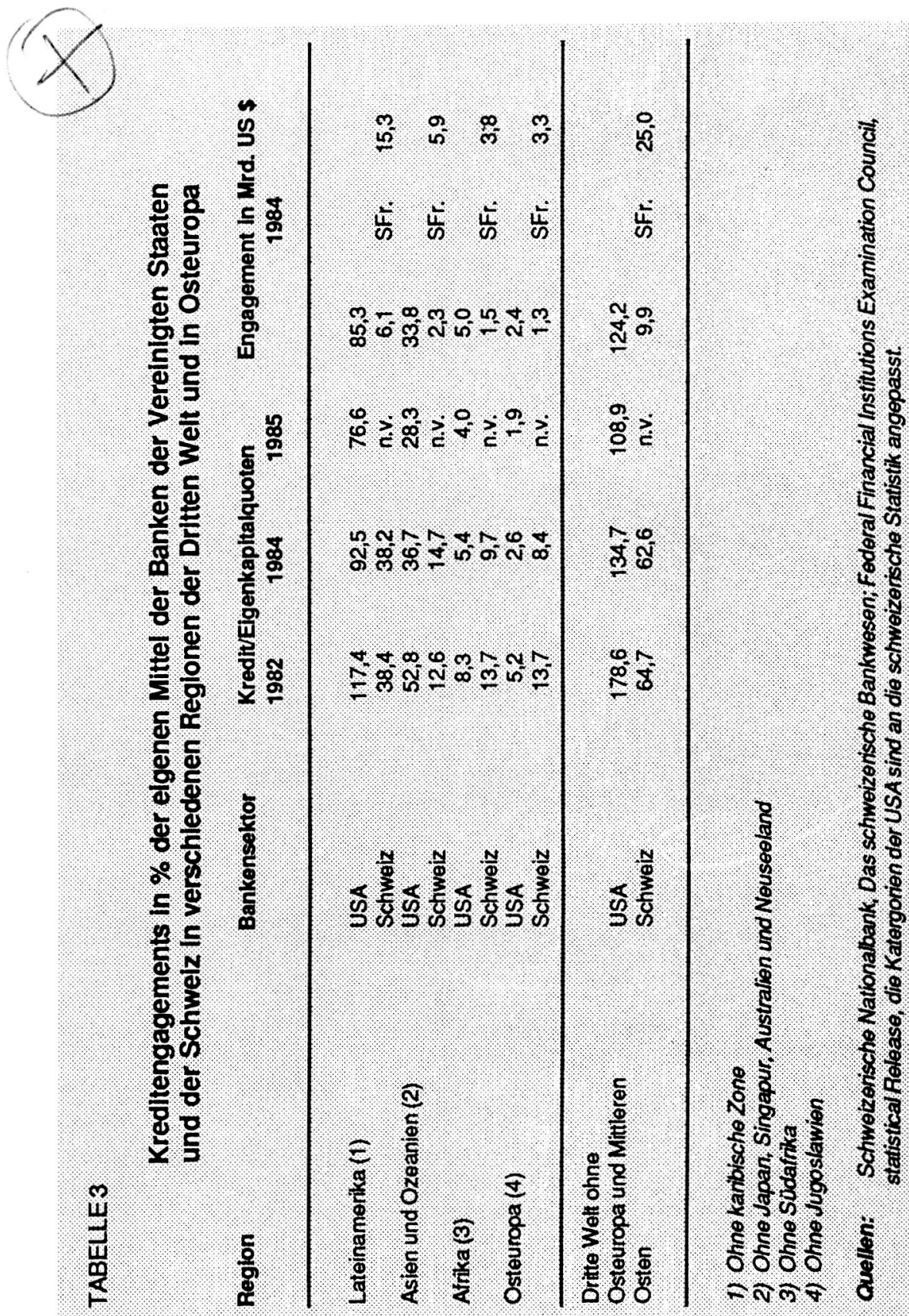


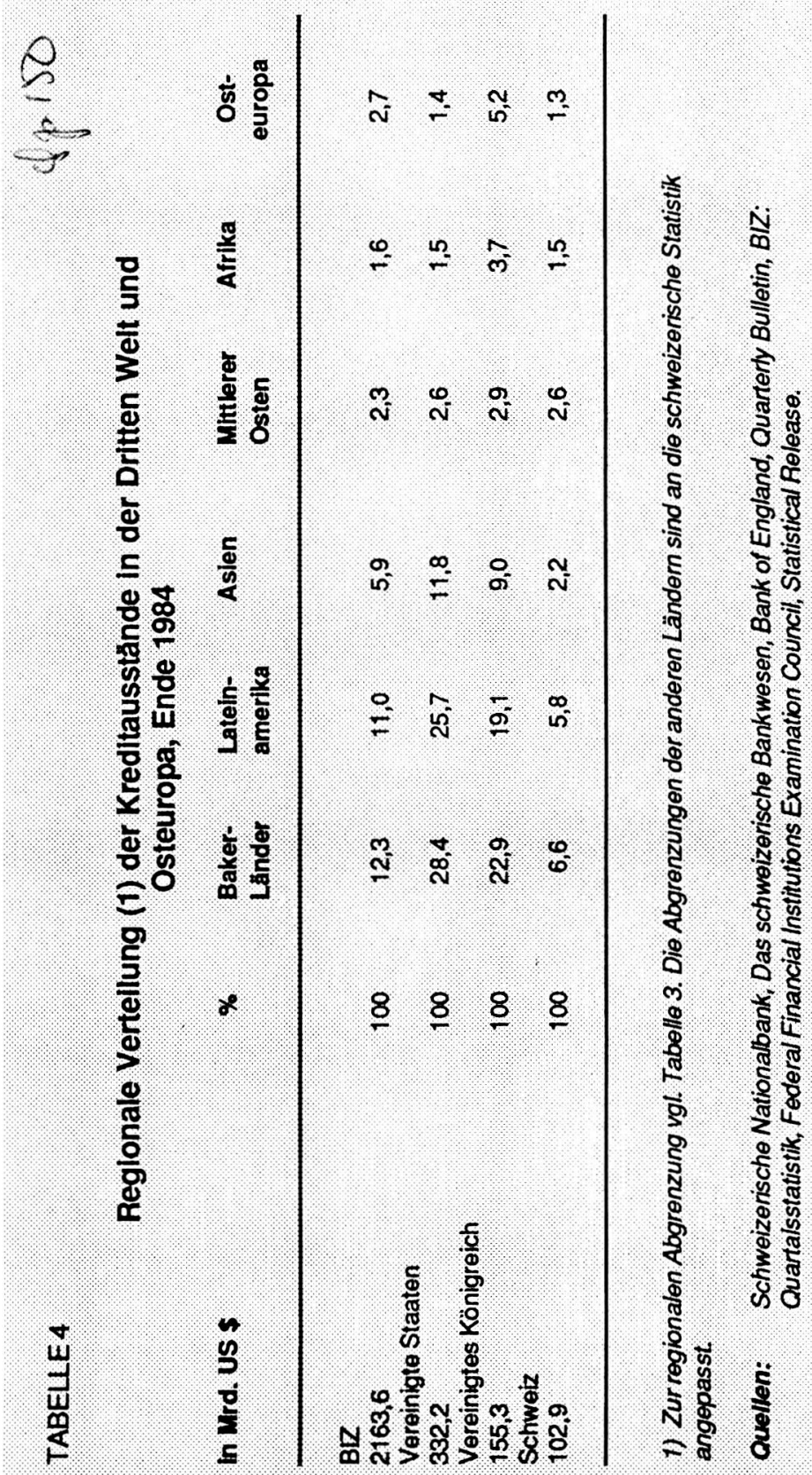




\section{Die Baker-Initiative und die Haltung der schweizerischen Banken}

Das Ergebnis des vierjährigen Krisenmanagements lässt sich wie folgt zusammenfassen:

- Das Krisenmanagement konnte bisher erfolgreich die Zahlungsunfähigkeit einer Reihe von Ländern verhindern. Insbesondere die in der zweiten Jahreshälfte 1982 und Anfang 1983 bestehende Gefahr, dass die Verschuldungskrise ausser Kontrolle geraten und das weltweite Handelsund Finanzsystem in Schwierigkeiten bringen könnte, scheint heute gebannt zu sein.

- Die externe Anpassung der Entwicklungsländer ist dank grosser wirtschaftspolitischer Anstrengungen rasch vorangeschritten. Damit ist der externe Finanzbedarf dieser Länder stark zurückgegangen.

- Die Risikosituation der international tätigen Banken hat sich entscheidend verbessert; sie sind heute besser als 1982 gegenüber Kreditausfällen gewappnet. Die schweizerischen Banken waren zu keinem Punkt in gefährlicher Weise direkt engagiert. Ihr Engagement in der Dritten Welt fällt nicht besonders ins Gewicht.

- Das Krisenmanagement hat aber in zwei Bereichen seine Ziele bisher nicht erreicht. Zum einen blieb das erhoffte Wirtschaftswachstum in den Schuldnerländern aus und dies hat die politischen und sozialen Spannungen in diesen Ländern in gefährlicher Weise erhöht. Zum anderen ist die Wiederherstellung der Kreditwürdigkeit der Entwicklungsländer in der Regel nicht gelungen, und folglich ist die Wiederaufnahme der freiwilligen Kreditvergabe ausgeblieben.

Angesichts dieser schwierigen Lage hatte im Herbst 1985 der amerikanische Finanzminister Baker auf der Tagung von Internationalem Währungsfonds und Weltbank der internationalen Schuldenproblematik einen neuen politischen Anstoss gegeben. Sein «Programm für ein tragfähiges Wachstum» basiert auf drei Elementen.

- Erstens werden die Schuldnerländer aufgefordert, eine marktorientierte Wirtschaftspolitik einzuleiten, um das Wachstum zu fördern, die Inflation zu beseitigen und die externen Ungleichgewichte weiter zu reduzieren. Zur Milderung des Konfliktes zwischen Wachstum und Anpassung empfiehlt das Programm, ein Wirtschaftswachstum über die Stärkung der Angebotskräfte zu bewirken.

- Zweitens sieht das Programm zur Unterstützung der strukturellen Anpassungs- und Wachstumspolitik einen Anstieg der Bruttoausleihungen der multilateralen Entwicklungsorganisationen, insbesondere der Weltbank, um 50\% über einen Zeitraum von drei Jahren vor.

- Drittens werden die Geschäftsbanken aufgefordert, in diesem Zeitraum Neuausleihungen in Höhe von 25 Mrd. US-Dollar bereitszustellen, was in etwa dem Beitrag der multilateralen Entwicklungsorganisationen entspräche. 
Die drei Elemente der Baker-Initiative sind eng miteinander verknüpft. Das vorgesehene Neugeld soll den Ländern Zeit einräumen, um ihre Volkswirtschaften auf einen neuen Wachstumspfad zu bringen. Umgekehrt ist die Anwendung einer marktorientierten Wachstumspolitik, die Beendigung der Kapitalflucht und die Repatriierung grösserer Teile des Fluchtkapitals die Voraussetzung für Neugeld. Das Zusammenspiel dieser Faktoren soll letzlich die Kreditwürdigkeit der Schuldnerländer wiederherstellen und die Wiederaufnahme der freiwilligen Kreditvergabe durch die Banken ermöglichen.

Trotz der Kritik an wichtigen Punkten ist das Programm im grossen und ganzen von den verschiedenen nationalen Bankengruppen einschliesslich der schweizerischen Banken begrüsst worden (10). Im Gegensatz zu dem kurzfristigen Krisenmanagement der Jahre 1982 bis 1985 visiert die BakerInitiative ein langfristiges Fundament zur Sanierung der Verschuldungslage an. Dabei anerkennt sie auch, dass den Regierungen der Industrieländer und insbesondere der Regierung der Vereinigten Staaten bei der Ueberwindung der Schuldenkrise eine Führungsrolle zukommt. Allerdings bleiben nach Ansicht vieler Banken gerade die Aufgaben der Industrieländer unpräzise definiert (11). Von Seiten der Banken, aber auch von anderen Stellen wird betont, dass der Erfolg der Baker-Initiative nicht zuletzt von den weltwirtschaftlichen Rahmenbedingungen abhängen wird, die durch die Regierungen der Industrieländer geschaffen werden. Die wichtigsten Voraussetzungen für eine dauerhafte Bewältigung der Verschuldungslage auf diesem Gebiet sind ein kontinuierliches Wirtschaftswachstum der Industrieländer, koordinierte Runden der Zinssenkung und offene Märkte für die Exporte der Entwicklungsländer. Eine kooperative Bewältigung der Schuldenkrise verlangt von den Regierungen der Gläubigerländer aber auch Beiträge im Finanzbereich, wie z.B. die Wiederbelebung der Exportrisikogarantie, mehrjährige Umschuldungsabkommen oder die Erhöhung der Kapitalbasis der Weltbank. Die Baker-Initiative lässt aber auch eine Reihe technischer Fragen offen, beispielsweise wie die neue Wachstumspolitik der Schuldnerländer implementiert und kontrolliert werden soll oder wie die Arbeitsteilung zwischen Weltbank und IWF aussehen soll. Auch die Frage nach der erweiterten Rolle der Weltbank und die Formen der Kooperation mit den Geschäftsbanken sind zum Teil noch ungeklärt (12).

Die Haltung der schweizerischen Banken ist bei verschiedenen Anlässen erläutert worden. Die Bereitschaft der amerikanischen Regierung, die internationale Schuldenkrise auch als ein politisches Problem anzuerkennen und anzugehen, wird allgemein begrüsst. Die prinzipielle Unterstützung der Baker-Initiative durch die schweizerischen Banken wurde verschiedentlich erklärt, gleichzeitig wurde aber auch darauf aufmerksam gemacht, dass die Beiträge der Banken von Fall zu Fall erfolgen würden. Betont wird auch die Bedeutung eines angemessenen Beitrages der Regierungen der Industrieländer (13).

F. Lütolf vom Schweizerischen Bankverein hat zudem wiederholt darauf hingewiesen, dass die Wiederaufnahme der freiwilligen Kreditvergabe und 
die Normalisierung der Kreditbeziehungen nicht immer wieder verschoben werden darf, wenn die Beziehungen zwischen den Gläubigern und Schuldnern nicht dauerhaften Schaden erleiden sollen (14). Wie bereits erwähnt wurde, vertiefen fortgesetzte Umschuldungsrunden und unfreiwillige Kreditzusagen bestehende Verzerrungen in den Kreditbeziehungen, insbesondere dann, wenn Teile der Schuld als notleidend angesehen werden müssen.

Um allmählich zu ehper Normalisierung der Kreditbeziehungen zurückzufinden, schlägt $F$. Lütolf) eine graduelle und selektive Wiedereinführung der freiwilligen Kreditvergabe und Erweiterung des Handlungspielraumes der Banken vor. Zu diesem Zweck sind Bereiche zu definieren, die sich für eine solche Normalisierung eignen. Die handelsbezogene Kreditvergabe bietet sich z.B. für eine begrenzte Wiederbelebung der freiwilligen Kreditvergabe an, zumal in den meisten Fällen Handelskredite ohnehin regelmässig bedient werden. Die reibungslose Gewährung von Handelskrediten ist für eine effiziente Abwicklung des Handelsverkehrs der Entwicklungsländer von besonderem Interesse. Gleichzeitig bietet diese Kreditart wegen ihrer Kurzfristigkeit und ihres handelsbezogenen Charakters den Banken mehr Sicherheit als reine Finanzkredite. Eine Wiederbelebung der freiwilligen Kreditvergabe in diesem Bereich brächte somit für beide Parteien Vorteile. Voraussetzung dafür ist allerdings, dass die Schuldnerländer sich verpflichten, in Zukunft Handelskredite präferenziell zu bedienen und sie von den Umschuldungsabkommen auszuschliessen.

Ein anderer Bereich, der sich für eine schrittweise Normalisierung eignet, betrifft die Wahl der Beitragsform, mit der sich die Banken an den Umschuldungsabkommen beteiligen. Die heute übliche Praxis, den Finanzbeitrag der Banken ausschliesslich in Form von Neugeld zu geben, wird als unnötiger Zwang empfunden. Es sollte jeder Bank freigestellt werden, zu bestimmen, ob sie ihren Beitrag als Neugeld gibt oder z.B. alternativ einen Zinsaufschub gewährt, solange der Nettoeffekt für den Schuldner der gleiche bleibt. Für viele Banken ist dieser zusätzliche Handlungsspielraum bedeutungsvoll, weil es innen dadurch erleichtert wird, die Formen der Risikovorsorge vorzunehmen, die sie als notwendig erachten.

Ein dritter Bereich, in dem sich der Entscheidungsspielraum der Banken vergrössern liesse, betrifft die Option, umstrukturierte Fälligkeiten in die Heimatwährung umdisponieren zu können. Auf diese Weise werden insbesondere für die europäischen Banken ihre Refinanzierungsmöglichkeiten besser mit der Struktur und Qualität ihres Kreditportefeuilles übereinstimmen. Um den individuellen Risikopräferenzen der einzelnen Banken in Zukunft wieder vermehrt Rechnung tragen zu können, sollte auch daran gedacht werden, dass die Banken auf die Dauer auch mehr Freiheit bei der Verteilung ihrer Gelder auf die Baker-Länder haben sollten, und Kreditverträge formuliert werden, die der wirtschaftlichen Leistungsfähigkeit eines Landes Rechnung tragen (15).

All diese Vorschläge haben eine langfristige Verbesserung und effizientere Gestaltung der Kreditbeziehungen zwischen den Geschäftsbanken und 
den hochverschuldeten Entwicklungsländern im Auge. Mehr Handlungsfreiheit und Flexibilität der Banken bei der Kreditentscheidung, die individuelle Bewertung der Länderrisiken und die Berücksichtigung unterschiedlicher Risikopräferenzen sind auf die Dauer unerlässlich für eine effiziente Kapitalallokation zwischen Nord und Süd (16). Dabei wird nicht übersehen, dass ein effizientes System der Kreditbeziehungen nicht unbedingt zu einem Ressourcentransfer führt, der auch gleichzeitig unter entwicklungspolitischen Gesichtspunkten als wünschbar und angemessen zu bewerten wäre. Dies ist jedoch kein Argument für eine fortgesetzte unfreiwillige Kreditvergabe. Letzten Endes wird der entwicklungspolitisch erwünschte Ressourcentransfer nur erreicht werden können, wenn a) das ausgeliehene Kapital marktmässig bewertet und honoriert wird, b) die Schuldnerländer eine verlässliche Wirtschaftspolitik betreiben und c) die offizielle Entwicklungshilfe noch verbleibende Finanzlücken schliessen hilft.

\section{FUSSNOTEN}

1. Beispielsweise liessen sich für Länder, deren Exporteinnahmen stark von Schwankungen einzelner Weltmarktpreise für Rohstoffe abhängen, Kreditverträge formulieren, die die Bedienung der Kredite von der Entwicklung der Weltmarktpreise für Rohstoffe abhängig machen. Vergleiche zu Vorschlägen dieser Art sowie einer Analyse systematischer Probleme in den derzeitigen Kreditbeziehungen zwischen Banken und Entwicklungsländern insbesondere. Lessard, D. (1983) und Swoboda, A.K. (1985).

2. Im Zusammenhang mit der Baker-Initiative werden im allgemeinen 15 Schuldnerländer genannt: Argentinien, Bolivien, Brasilien, Chile, Ecuador, die Elfenbeinküste, Jugoslawien, Kolumbien, Marokko, Mexiko, Nigeria, Peru, die Philippinen, Uruguay und Venezuela.

3. Vgl. IWF, World Economic Outlook, April 1986.

4. Cline, William R. (1983), Tabelle 6.

5. Dennoch dürfte das schweizerische Kreditengagement zwischen 1982 und 1984 in der Dritten Welt leicht zurückgegangen sein, jedoch wird dies durch den Wechselkurseffekt des starken Dollars überspielt, der dazu führte, dass die in Schweizer Franken ausgedrückten Dollarausstände Ende 1984 höher bewertet wurden als Ende 1982.

6. Internationale Vergleiche der Kredit/Eigenkapitalquoten und Engagements sind nur unter starken Vorbehalten möglich. Die Definition der eigenen Mittel und Formen der Rückstellungen variieren stark von Land zu Land. Ein Vergleich der US-Engagements mit denjenigen der Schweiz wird zudem durch die unterschiedliche Erfassung der grenzüberschreitenden Ausleihungen erschwert. Die US-Engagements sind auf Konzernebene konsolidiert und enthalten demnach korrekterweise nicht die grenzüberschreitenden Engagements der Banken an ihre eigenen Niederlassungen und Tochtergesellschaften im Ausland. Zudem sind sie nach dem Risikodomizil erhoben, d.h. sie werden um Bürgschaften und Garantien dritter Parteien korrigiert. Die schweizerischen Zahlen beinhalten demgegenüber nicht die grenzüberschreitenden Engagements ihrer Tochtergesellschaften im Ausland und werden nach dem Schuldnerdomizil erfasst. Eine nicht veröffentlichte Sondererhebung der Eidgenössischen Bankenkommission vom Frühjahr 1983 hat aber gezeigt, dass die hier verwendeten Zahlen der Schweizeri- 
schen Nationalbank über die Kreditengagements der schweizerischen Banken die "effektiven Grössenordnungen relativ gut wiedergeben» (Schweizerischer Bundesrat, 1984, S. 19). In demselben Bericht wird festgehalten, dass der Gesamtumfang des Länderengagements per Ende 1982 in über 60 Problemländern auf konsolidierter Basis und gemäss Risikodomizil $23 \mathrm{Mrd}$. SFr. ausmachte, was um einiges weniger ist als die eigenen Mittel der schweizerischen Grossund Auslandsbanken. Dieses Ergebnis ist konsistent mit unseren eigenen Berechnungen.

7. Banken, die ihre grenzüberschreitenden Positionen der Bank für Internationalen Zahlungsausgleich (BIZ) berichten. Dazu gehören die Banken der Zehnergruppe, der kleineren Industrieländer und der wichtigsten Offshore-Plätze.

8. Für eine ausführliche Diskussion über das Engagement und Verhalten der Geschäftsbanken ausgewählter Industrieländer in der Dritten Welt siehe Nunnenkamp, P. und G. Junge (1985).

9. World Bank, World Development Report 1986, Tabelle 2.12.

10. Vgl. dazu z.B. Neue Zürcher Zeitung 16.12.85. «Prinzipielles Plazet der Banken zum Baker-Plan» und am 30.1.1986 «Schweizer Antwort auf den Baker-Plan».

11. Ebenda

12. Vgl. dazu Kienberger, U., A. Calame und G. Junge (1986).

13. Vgl. dazu z.B. Handelsblatt, 19.11.1985, «Erfolg des Baker-Plans hängt auch von Kreditbereitschaft der Regierungen ab» und Basler Zeitung 18.12.85, "Schulden nicht mehr das dringendste Problem", Interview mit Hans J. Mast, SKA. Neue Zürcher Zeitung 16.12.85 "Prinzipielles Plazet der Banken zum Baker-Plan» und die Meldung vom 30.1.86, «Schweizer Antwort auf den Baker-Plan».

14. Lütolf, F. (1986a) und (1986b).

15. Im Falle Mexikos kann dies z.B. bedeuten, die Rückzahlung der Schulden des Landes an die Entwicklung des Oelpreises zu knüpfen. Siehe dazu Lütolf (1986b).

16. Für prinzipielle Diskussionen über die Bedingungen für effiziente internationale Kreditbeziehungen, siehe insbesondere Swoboda, A.K. (1985), Lessard, D. (1983) sowie Nunnenkamp, P. und G. Junge (1985).

\section{QUELLEN- UND LITERATURVERZEICHNIS}

Bank of England, Quarterly Bulletin, verschiedene Jahrgänge.

Bank für Internationalen Zahlungsausgleich, Quartalsstatistik, verschiedene Jahrgänge.

Cline, William R. (1983) International Debt and the Stability of the World Economy, Institute for International Economics, 4.

Federal Financial Institutions Examination Council, Statistical Release, verschiedene Jahrgänge.

Handelsblatt, 19.11.1985.

IWF (1986) Occasional Paper 43.

IWF (April 1986) World Economic Outlook.

Kienberger, U., A. Calame und G. Junge (1986), Baker-Initiative: Do the Numbers Add up? Prospects 1/1986, Swiss Bank Corporation, Basel.

Lessard, D. (1983), North-South. The Implications for Multinational Banking, Journal of Banking and Finance, 7.

Lütolf, F. (1986a), A Swiss Banker's View, The Baker-Initiative, Prospects No. 1, Swiss Bank Corporation.

Lütolf, F. (1986b), Die Rolle der Banken in der Schuldenkrise, Neue Zürcher Zeitung, 9.9.1986. 
Mast, J. (SKA), Schulden nicht mehr das dringendste Problem, Basler Zeitung, 18.12.1985.

Neue Zürcher Zeitung, 16.12.1985, 30.1.1986, 12.6.1986.

Nunnenkamp P. und G. Junge (1985), Die Kreditbeziehungen zwischen westlichen Geschäftsbanken und Entwicklungsländern. Unternehmerisches oder gesellschaftliches Risiko. Weltforum Verlag.

Schweizerischer Bundesrat, Bericht über die Risiken der internationalen Verschuldung, 12. März 1984.

Schweizerische Nationalbank, Das schweizerische Bankwesen, verschiedene Jahrgänge.

Swoboda, A.K. (1985), Debt and the Efficiency and Stability of the International Financial System, in Smith G.W. and J.T. Cuddington. International Debt and the Developing Countries, A World Bank Symposium, Washington, D.C.

World Bank, World Development Report 1986. 